\title{
Familial Hemophagocytic Lymphohistiocytosis
}

National Cancer Institute

\section{Source}

National Cancer Institute. Familial Hemophagocytic Lymphohistiocytosis. NCI Thesaurus. Code C61276.

A rare, life-threatening disorder usually appearing during the first few months of life. It is caused by abnormalities in the PRF1, UNC13D, and STX11 genes. It is characterized by histiocytic proliferation and phagocytosis. Patients present with fever, lymphadenopathy, and hepatosplenomegaly. 